\title{
On the acquisition of maximality in free relative clauses and plural definite descriptions*
}

\author{
Ivano Caponigro \\ University of California, San Diego \\ Neon Brooks \\ University of Chicago
}

\author{
Lisa Pearl \\ University of California, Irvine \\ David Barner \\ University of California, San Diego
}

\begin{abstract}
Plural definite descriptions (e.g. the things on the plate) and free relative clauses (e.g. what is on the plate) have been argued to share the same semantic properties, despite their syntactic differences: both are non-quantificational expressions referring to the maximal element of a given set (e.g. the set of things on the plate). Experimental support for this semantic analysis is provided by the first investigation ever of children's interpretation of both constructions. A Truth-Value Judgment task, an Act-Out task, and a corpus study of children's linguistic input show that children are aware that the two constructions are different from quantificational nominals (e.g. all the things on the plate, some of the things on the plate) very early on (4 years old), despite the major difference in frequency in the input. Children acquire the adult interpretation of both constructions at the very same time, around 6-7 years old. We suggest that this relative delay depends on children's difficulties with the concept of the maximal element of a set or its association with specific linguistic constructions.
\end{abstract}

Keywords: free relative clauses, plural definite descriptions, maximality, acquisition, experimental, Act-Out task, Truth-Value Judgment task, corpus analysis

\section{Introduction}

If there are six cookies in a box and nothing else, adult English speakers judge both sentences (1) and (2) true. The very same sentences are judged false in a situation in which there are only marbles in the box. If the box contains three marbles and three cookies, then (1) and (2) are either false or infelicitous/awkward.

(1) [The things in the box] are edible.

* We would like to thank Jennifer Audet, the audience at SALT 2010, the members of SemBabble at UCSD, and the members of LadLab at UCSD. 
On the acquisition of maximality

(2) [What is in the box] is edible.

More generally, sentences (1) and (2) have the same felicity/truth-conditions ${ }^{1}$, even though they differ syntactically in their bracketed subject constituents. In (1), the bracketed subject is a plural definite description (PDD), i.e., a nominal expression introduced by the (syntactically a DP with the determiner the as its D head and an NP as its complement ${ }^{2}$ ). In (2), however, the bracketed subject is a free relative clause (FR), i.e., a clause introduced by a $w h$-word that is not interpreted as a question (syntactically a non-interrogative embedded $w h-\mathrm{CP}^{3}$ ).

In the past forty years, the semantic properties of PDDs have been carefully described and several accounts have been suggested. Though different in the details, all the accounts agree on at least two main properties of PDDs. First, a PDD is referential rather than quantificational. In other words, a PDD semantically behaves more like a proper name in referring to a specific object rather than quantifying over a set of objects in the way quantified nominals like every cookie or all the cookies $d o^{4}$. Second, a PDD does not refer to any random object in a given set, but only to the (formally definable unique) maximal element of that set. Different specific proposals may vary on the nature of the maximal object a PDD refers to: a (possibly plural) maximal individual out of a set of (atomic and plural) individuals or a maximal set of individuals out of a set of sets of individuals ${ }^{5}$.

The very same semantic approach has then been extended to FRs ${ }^{6}$. Thus, both PDDs and FRs have been analyzed as referential expressions "triggering maximality" (i.e., referring to the maximal element of a given set). However, the two constructions

$1 \mathrm{We}$ are abstracting away from the case in which there is only one object in the box. In such case, sentence (1) feels awkward, since the plural definite description seem to license the inference (a presupposition? an implicature?) that there must be more than one thing in the box. On the other hand, sentence (2) feels fully acceptable in this situation as well. The difference may be related to the fact that in English definite descriptions can be syntactically singular or plural, while free relatives are always syntactically singular, even when they are semantically plural, i.e., they refer to a plural individual.

2 More articulated internal structures have been suggested for PDDs. See Bernstein 2003 and Longobardi 2003 for an overview of DPs, their internal structures, and supporting evidence.

3 See van Riemsdijk 2005 for a thorough survey.

4 See Löbner 2000: 233-234, 251-253 and references therein for a discussion of the non-quantificational nature of PDDs and definite descriptions in general.

5 See Abbott 2010 for an accessible overview of the main facts and proposals about definiteness and definite descriptions in general and PDDs in particular; Schwarzschild 1996 and Landman 2000 for more technical presentations of the issues about PDDs and for specific proposals according to which PDDs refer to maximal sets of individuals rather than maximal individuals; Link 1983 for the fully detailed version of the specific semantic analysis of PDDs we are adopting according to which PDDs refer to maximal individuals rather than maximal sets.

6 See Jacobson's 1995 seminal work and, among others, Rullmann 1995, Dayal 1996, and Caponigro 2003, 2004). 
differ in their syntax - as briefly sketched above - and therefore in their syntaxsemantics mapping, as well. Maximality in PDDs is usually considered to be triggered overtly by the determiner the (e.g., Link 1983). Maximality in FRs, instead, has been argued not to be due to any overt lexical item, but to result from the set of type-shifting operations that have been invoked for (bare) nominals ${ }^{7}$, with a silent operator, sister of the $\mathrm{CP}$ of the FR, acting as the trigger of the shifting (Caponigro 2003, 2004).

Left uncertain by such analyses is whether these two expressions of maximality are attributable to a common semantic operator - overtly expressed in the first case, and covertly in the second - or whether the two operators are represented by distinct mental types (i.e., are psychologically distinct). These two alternative representational accounts are difficult to distinguish on the basis of descriptive adequacy. However, they make different predictions for language acquisition, the focus of the present study. Here we explore the course of PDD and FR acquisition in 4- to 7-year-old children, and argue that the developmental data lend support to the idea that PDDs and FRs have a common semantic representation.

Although PDDs and FRs have ostensibly similar interpretations, they differ substantially both in their form, and, as we will show, their frequency in language input to children. Given these grammatical and distributional differences, we might expect corresponding differences in the rate at which children acquire each type of expression. For example, we might expect that children interpret PDDs - e.g., the things on the plate - as maximal earlier than for FRs - e.g., what's on the plate. Another possibility, consistent with the hypothesis that these expressions share a common semantic representation, is that PDDs and FRs emerge in synchrony despite their surface differences. Although either semantic account could explain a non-synchronous emergence of PDDs and FRs in acquisition - due to differences in frequency, syntactic complexity, etc. - a synchronized emergence would be most naturally explained by the hypothesis that both forms depend on a common semantic operator, whose emergence permits both forms to be acquired.

Previous acquisition studies (Munn, Miller \& Schmitt (2006) for PDDs and Modyanova \& Wexler (2008) for FRs) have not yet established when children acquire adult-like interpretations of these forms, or how they are related in acquisition. Also, previous studies have used different methods to investigate the acquisition of PDDs and FRs, making it difficult to compare previous results.

We assess children's understanding of both constructions using the same set of tasks to test the hypothesis that (i) PDDs and FRs are mapped onto the same meaning, (ii) the meaning they are mapped onto is the maximal element of a given set, and (iii) that these meanings emerge at the same time in acquisition. We also investigate

7 See Partee 1986, Chierchia 1998, and Dayal 2004, among others. 
On the acquisition of maximality

the frequency of both forms in child-directed speech. Our findings show that from very early on children treat the two constructions as semantically equivalent, but as semantically different from quantificational nominals.

We also find that children map both PDDs and FRs onto maximal elements at around the same time, but do so relatively late (when they are 6 to 7 years old). These results are particularly interesting given our analysis of child-directed speech, which finds that PDDs and FRs differ massively in their frequency in children's input. Together, our findings support the hypothesis that a common semantic mechanism supports children's maximal interpretations of both PDDs and FRs.

The paper is structured as follows. Section 2 briefly reviews previous related acquisition studies. Section 3 presents and discusses the results of our two experiments on the acquisition of PDDs and FRs: a Truth-Value Judgment task and an Act-Out task. Section 4 presents and discusses the findings of our corpus study in which we look at child-directed speech containing PDDs and FRs. Section 5 concludes with some general remarks and open issues.

\section{Previous acquisition studies}

In this section, we briefly review the few previous studies about children's interpretation of PDDs and FRs. We are aware of only one study of PDDs and another one regarding FRs. We discuss them in turn below.

Munn et al. (2006) investigate the acquisition of PDDs together with singular definite descriptions and indefinite DPs in English and Spanish by means of an Act-Out task. To test the interpretation of English PDDs, they tested 15 children (aged 3;0 to 5;5, mean 4;1) and presented scenarios with toys such as one where three frogs are next to a barn and three frogs are next to a house. They then uttered the following request (where the PDD is italicized): "Give me the frogs next to the barn."

Almost all children provided adult-like responses (95\%), and gave all three frogs next to the barn, i.e., they give the maximal element of the relevant set of frogs (44 adult controls selected the 3 frogs $100 \%$ of the time). Munn et al. (2006) conclude that most children interpret PDDs correctly, i.e., maximally, and do so by at least the age of 3. However, some issues temper this conclusion. First, and most critically, the study did not include control trials to be sure that children would not select the maximal set for other requests - e.g., "Give me some of the frogs next to the barn". Once young children have begun collecting frogs, they may see no reason to stop at two, the minimum required by the plural noun. Second, Munn et al.'s calculation of $95 \%$ success did not include children who only gave one item, which is quite clearly a failure to comprehend the maximality requirements of the PDD. When these children are included, the total is now $85 \%$. Finally, no breakdown by age 
is provided, so it is hard to see the progression of acquisition. It still needs to be determined at which age - if any - children do not understand maximality in PDDs.

In a separate study, Modyanova \& Wexler (2008) assess children's interpretation of FRs by means of a Truth-Value Judgment (TVJ) task ${ }^{8}$. Children were shown pictures such as one in which two green apples and one red apple are under a blanket that is partially lifted so that children can see under it, while another red apple is completely outside the blanket.

Children were then asked a question containing a FR - e.g., "Is what is under the blanket red?" The authors expected the correct answer to be "no". The FR what is under the blanket refers to the plural individual made of two red apples and one green apple. As such, this plural individual is not red, since an atomic part of it is green. Instead, most children replied "yes", regardless of age ("no" answers: 16 3- to 5 -year-olds $=17 \% ; 13$ 6- to 8 -year-olds $=22 \%$; 9 9- to 12 -year-olds $=33 \%$ ). Crucially, most adults also did not answer "no" as predicted (22 18-24-year-olds=30\%). Therefore, based on this task, children do not interpret FRs as maximal - but neither do adults. This result is problematic in light of speakers' intuitions about the meaning of FRs in many other contexts, as discussed in section 1.

Although it is possible that FRs do not actually receive a maximal interpretation, a more likely explanation of Modyanova \& Wexler's data is that the contexts used in their TVJ task violate what Löbner (2000) and Gajewski (2005) call the presupposition of homogeneity. When a predicate like be red - which Löbner (2000) calls "summative" - applies to a PDD (or any other expression referring to a plural individual), it requires that all of the atomic individuals that comprise the plural individual be red for the sentence to be true. Similarly, it requires that none of the atomic individuals be red in order for the sentence to be false. In any other situation (e.g., some of the atomic individuals are red and some are green), the sentence does not receive a truth-value. Thus, by this account, if speakers are forced to assign a truth-value anyway, they may base responses on factors that are not related to the semantics of maximality.

In conclusion, although two previous studies have independently examined children's understanding of maximal expressions - PDDs and FRs - they have done so using two different methods, each of which presents difficulties of interpretation. It is therefore difficult to determine when the respective forms are acquired, and whether they exhibit similar patterns of acquisition. One of our goals is to provide the first simultaneous study of both PDDs and FRs using the same set of methods, to allow a direct comparison of results. To do so, we tested children using both the Truth-Value Judgment and Act-Out tasks. Another goal was to provide something that is still missing from the studies above: an assessment of the age at which

8 See Flynn \& Foley 2004 for children's acquisition of FRs as syntactic objects. 
On the acquisition of maximality

children acquire maximality associated with PDDs and FRs.

\section{Experiments}

\subsection{Experiment 1: Truth-Value Judgment Task}

The purpose of our first experiment was to assess children's maximal interpretations of PDDs and FRs using a task similar to that of Modyanova \& Wexler's (2008) task. We introduced a character (Cookie Monster) who loves cookies but strongly dislikes onions, and asked children questions such as "Does Cookie Monster like the things on the plate?" or "Does Cookie Monster like what's on the plate?". We contrasted children's interpretation of PDDs and FRs with control items, including the quantified nominals some of the things and all the things, and the numeral one e.g., "Does Cookie Monster like one of the things on the plate?".

\subsubsection{Methods}

Participants. We tested 69 children ages 4 to 7 . There were 194 -year-olds $(\mathrm{m}=4 ; 7$, range: 4;0-4;11), 17 5-year-olds $(\mathrm{m}=5 ; 8$, range: 5;1-5;11), 20 6-year-olds $(\mathrm{m}=6 ; 5$, range: $6 ; 0-6 ; 11)$, and 13 7-year-olds $(\mathrm{m}=7 ; 4$, range: 7;1-7;8). Families were recruited by phone or through daycares in the greater San Diego area. We also tested sixteen undergraduates at The University of California, San Diego, who participated for course credit.

Materials and Procedure. The experimenter first presented participants with a color picture of Sesame Street's Cookie Monster. Participants were asked, "Do you know who this is? This is Cookie Monster! Do you know what Cookie Monster loves to eat?" When the participant responded "cookies", the experimenter presented a picture of a single chocolate chip cookie and said, "That's right! Cookie Monster loves cookies. Here is a cookie!" The experimenter also told participants, "Do you know what Cookie Monster really does not like? Cookie monster does not like onions," and children were shown a picture of an onion. Children were asked to label both the cookie and the onion, and identify which food Cookie Monster liked, and which he did not like. Once the experimenter was confident that a child could identify both cookies and onions and knew Cookie Monster's preferences, he or she began the experimental trials.

Participants were given 15 trials. On each trial the experimenter presented a picture of a plate with 6 objects (all cookies, all onions, or half cookies and half onions) in front of the child. For each picture, participants received 5 different question trials: a FR trial (“Does Cookie Monster like what's on this plate?"), a PDD 
trial ("Does Cookie Monster like the things on this plate?"), two quantifier control trials ("...all the things..." and "...some of the things...") and one numerical control trial ("...one of the things..."). Trials were presented in two quasi-random orders, with no two trials occurring consecutively in which the same picture was shown or the same question was asked.

\subsubsection{Results}

For control trials, children and adults responded similarly. Across questions and age groups, children correctly responded "no" to $97.3 \%$ of questions about the plate with 6 onions, while adults said "no" $95 \%$ of the time. Similarly, children correctly replied "yes" to $86.1 \%$ of questions about the plate with 6 cookies, while adults said "yes" $90 \%$ of the time. Repeated measures ANOVAs of each plate type showed no main effect of age ( $p$ s $>0.05)$ and no interaction of age and question type ( $p s>0.1)$. The remaining analyses focus on the critical mixed plate trials.

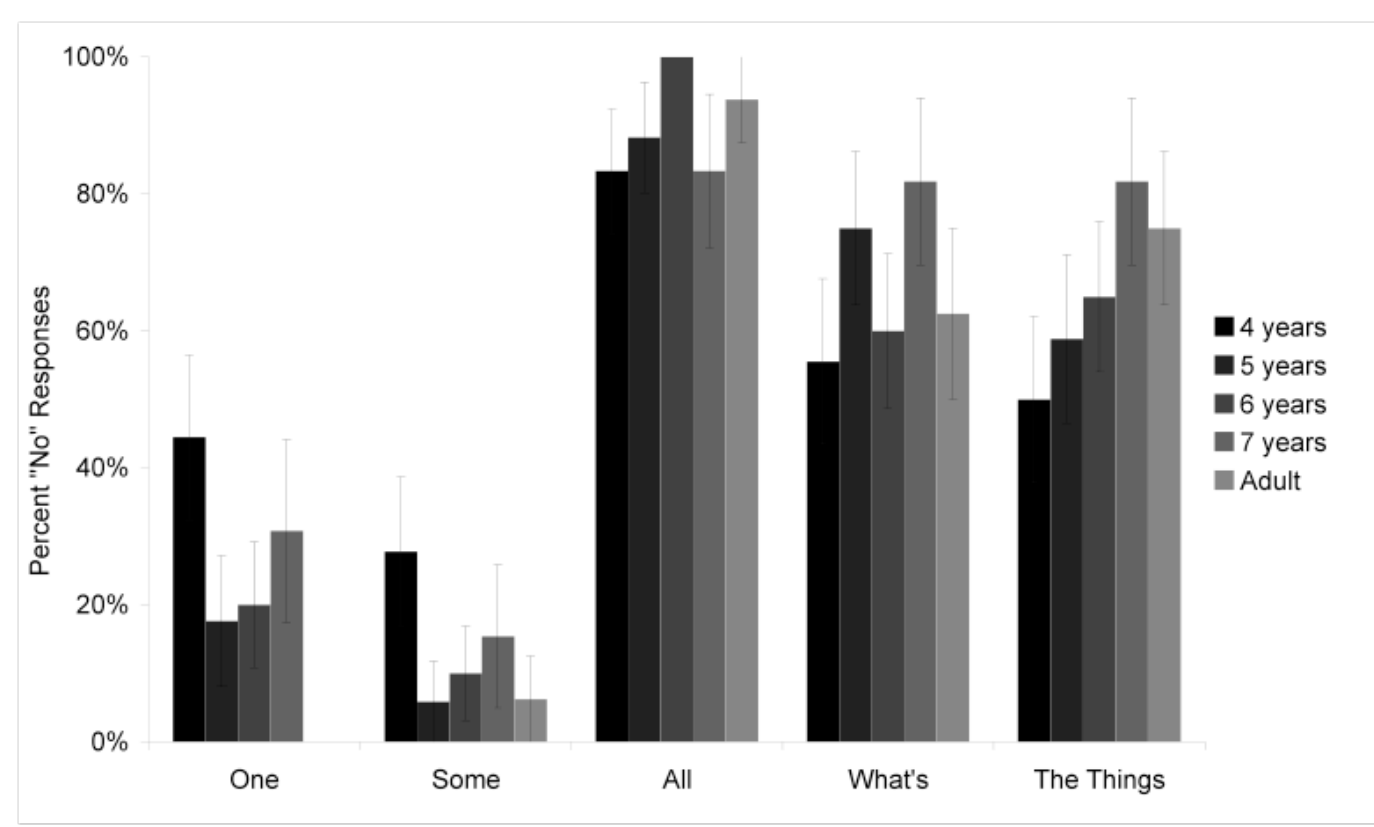

Figure 1 Percent of participants in each age group who said "no" to each question on the Truth-Value Judgment task. Error bars represent standard error.

Figure 1 shows participants' responses to critical trials, on which mixed sets were presented (i.e., cookies and onions). A 5(Question Type) X 5(Age Group) repeated measures ANOVA found a significant main effect of Question Type, $(F(4,304)=$ 
$70.1, p<0.001)$, but no main effect of Age Group $(p=0.778)$. There was also a significant interaction between Question Type and Age Group $(F(16,304)=2.22$, $p<0.01)$. To explore the main effect of Question Type we conducted TukeyHSD post hoc tests, and found significant differences between all question types ( $p$ s $<.01)$, except for one vs. some $(p=.56)$ and PDDs vs. FRs $(p=.99)$. This suggests that, overall, FRs and PDDs were interpreted maximally more frequently than DPs with the determiners one or some, but less frequently than those with all. However, participants' responses to questions with FRs and PDDs were not different from chance in most cases, making it unclear whether performance reflected true knowledge or random guessing. On FR trials, only 5-year-olds and 7-year-olds were statistically better than chance (1-sample t-tests, all $p \mathrm{~s}>0.05$, except for 5-year-olds, $t(15)=2.24, p<0.05$; and 7-year-olds, $t(10)=2.61, p<0.05)$. On PDD trials, only 7-year-olds $(\mathrm{t}(10)=2.61, p<0.05)$ and adults $(t(15)=2.24, p<0.05)$ were statistically above chance. We next explored the interaction of Question Type and Age Group. Univariate ANOVAs conducted on each Question (with Age Group as a between subjects factor for each analysis) revealed that this interaction was mediated by an effect of Age that is only present for the one question $(F(4,83)=2.79$, $p<0.05$; all other $p \mathrm{~s}>0.05)$. Thus, there was no evidence of age-related change in participants' responses to the critical FR and PDD trials, despite the appearance of an age-related trend in the case of the PDD.

\subsubsection{Discussion}

These data suggest that, beginning as young as 4 years of age, children do not treat FRs and PDDs the same as quantifiers like some. On the other hand, even adults fail to treat FRs and PDDs as equivalent to all on this task. As with Modyanova \& Wexler's (2008) task, it is difficult to assess children's performance in light of adults' lower-than-expected performance on the task. Additionally, as acceptance rates hover just above 50\% for the critical trials until age 7, it is difficult to determine whether younger children are interpreting these sentences maximally half the time, or whether they are simply guessing.

The imperfect performance of adults on this task may reflect the issue discussed in section 2 regarding the TVJ task of Modyanova \& Wexler (2008). As in their study, a violation of the presupposition homogeneity may have been triggered by an interaction between a summative predicate and its plural individual denoting argument, as argued by Löbner (2000) and Gajewski (2005). If the verb like that we used in our experiments is summative with respect to its object argument which was realized by a PDD or a FR in our test sentences - then the presupposition would require Cookie Monster to like all the things on the plate or none of things in

order for the answer to be question to be "yes" or "no" respectively. In any other 
circumstance, including our experimental conditions, the presupposition might be violated, such that no true or false answer can be given to the question. Thus, our results are consistent with the conclusion that a TVJ task does not offer a valid test of maximality, since any critical condition that is capable of assessing maximality will necessarily involved mixed sets in which the presupposition of homogeneity is violated. Based on this analysis, Experiment 2 investigated a similar group of children with PDDs, FRs, and control items using an Act-Out task.

\subsection{Experiment 2: Act-Out Task}

Experiment 2 tested children using an Act-Out task in which children were instructed to give a set of objects to the experimenter. This task provides a more conservative test of interpretation as it requires a child to interpret the sentence and to generate sets accordingly, rather than asking them to choose between two denotations (neither of which may actually correspond to the child's own semantic interpretation). Additionally, we tested children with control trials including quantifiers (some, all) and nonsense words (blick) in order to determine whether children's behaviors reflected true knowledge or merely random responding.

\subsubsection{Methods}

Participants. We tested a total of 67 children between 4 and 7 years of age. There were 13 4-year-olds ( $\mathrm{m}=4 ; 8$, range: 4;1-4;11), 19 5-year-olds $(\mathrm{m}=5 ; 5$, range: $5 ; 0-5 ; 11), 18$ 6-year-olds $(\mathrm{m}=6 ; 5$, range: 6;2-6;11) and 17 -year-olds $(\mathrm{m}=7 ; 5$, range: $7 ; 0-7 ; 11)$. Children were recruited by phone or through daycares in the greater San Diego area to participate in this task. An additional 17 children were tested but excluded from analyses for failure to complete the task (2), for giving the same number of items on all trials (2), and for failing to give 1 item when asked for one on control trials (13). We also tested 16 University of California San Diego undergraduates, who participated for course credit. Children or adults who participated in Experiment 1 were not eligible for Experiment 2.

Materials and Procedure. The experimenter placed a plastic sand bucket and a colorful paper plate in front of the participant, and placed four pieces of plastic fruit (an orange, an apple, a banana, and a strawberry) in each of the two locations. Participants were told, "In this game, I'm going to ask you to give me food from the plate (experimenter points to plate) OR from the bucket (experimenter points to bucket). Listen to what I ask for, and then put the food in my hands. My eyes will be closed, so when you're done giving me the food, say, 'I'm done!' " Children were then asked to identify the plate and the bucket. After they had identified both items 
On the acquisition of maximality

correctly, the experimenter began the test trials. Each child was given 12 trials. Each trial began with the experimenter making a request, and ended when the participant said she or he was done. There were six trial types: FR trials, in which participants were asked, "Can you give me what's in bucket/on the plate?", PDD trials, in which participants were asked, "Can you give me the things in the bucket/on the plate?", and Control trials, in which participants were asked for "one of the things", "some of the things", "blick of the things", or "all the things" from either the bucket or the plate. Each request type was performed on two trials: once for the plate and once for the bucket. Trials were presented in two quasi-random orders, with the same request never asked on consecutive trials and the same location never requested on more than two consecutive trials.

Consistent with our concerns regarding the Munn et al. (2006) study, pilot results showed that children who received a one trial very early in the experiment were less likely to give all fruit from the requested location on every trial. For this reason, the first trial in both orders was "Can you give me one of the things on the plate?" Children received neutral feedback throughout the experiment, except after trials where they gave fruit from the wrong location. In this case, the experimenter reminded the child to give food from the location requested. Trials in which children gave fruit from the wrong location were not repeated, but were excluded from analyses.

\subsubsection{Results}

Figure 2 shows the percentage of trials on which participants interpreted each request maximally (by giving all four items in the requested location). Since children who did not give one object when asked for "one" were excluded from the study, data for one trials were not included in analyses. A 5x5 repeated measures ANOVA was conducted, with Question Type as a within-subjects factor and Age Group as a between-subjects factor. The analysis revealed significant main effects of Question Type $(F(4,304)=247.4, p<0.001)$ and Age Group $(F(1,76)=780.4, p<0.001)$, and a significant interaction between Question and Age Group $(F(16,304)=13.7$, $p<0.001)$. Tukey-HSD post hoc tests found that, when all age groups were combined, all question types were different from each other $(p s<.0001)$, except for blick vs. some $(p=.64)$ and PDDs vs. FRs $(p=.85)$. One-way ANOVAs found no differences between age groups for blick or all trials, but found that maximal responses decreased with age for some (driven by a difference between 4-year-olds and all other groups) and increased significantly for FRs and PDDs. For PDDs, responses of adults, 6- and 7-year-olds were not significantly different from each other but the responses of each group differed significantly from those of 4- and 5 -year-olds $(p s<0.05)$. The difference between 4 - and 5-year-olds was not signifi- 


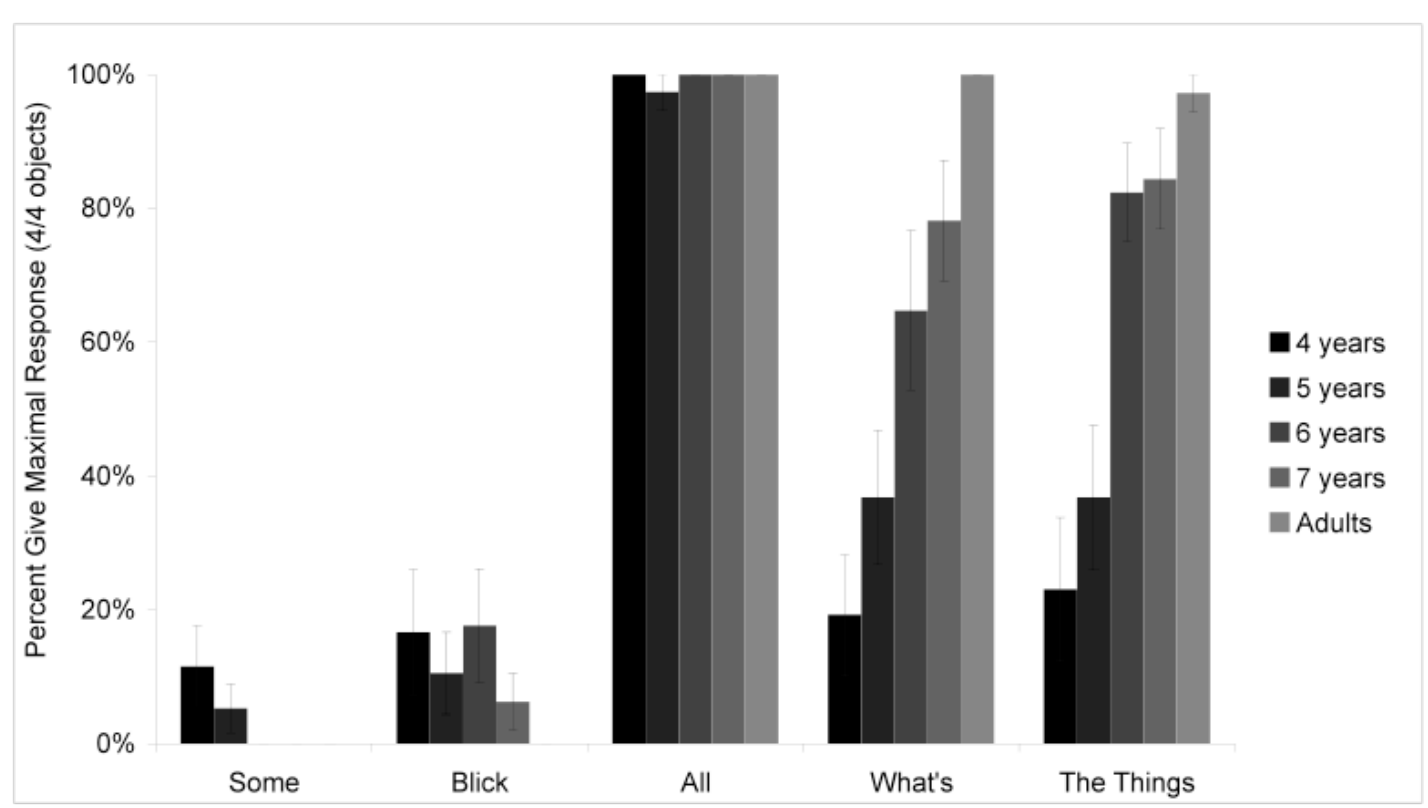

Figure 2 Percent of participants who give all objects for each request on the Act-Out task, by question and age group. Error bars represent standard error.

cant. On FR trials, adults and 7-year-olds did not differ from each other but differed from 4-, 5-, and 6-year-olds ( $p s<0.05)$.

\subsubsection{Discussion}

Results from the Act-Out task indicate a developmental progression in which young children (4- and 5-year-olds) do not initially interpret FRs and PDDs maximally, but begin to do so by 6 or 7 years of age, at which point their responses are similar to those of adults. The strong correlation between responses for these two expressions suggests that children acquire maximal interpretations for both expressions around the same time.

Children and adults were much less equivocal in their interpretations of FRs and PDDs in the Act-Out task than the TVJ task. While the TVJ results suggested that even adults do not always interpret FRs and PDDs maximally, the Act-Out task clearly shows that in a situation where they are prompted to act on their interpretation, adults and older children have a strong preference for the maximal interpretation. 
On the acquisition of maximality

\section{Children's exposure to maximal expressions: a corpus analysis}

Our experimental results suggest that young children do not initially believe that PDDs and FRs refer to maximal individuals. Instead, this knowledge emerges for both expressions simultaneously around ages 6-7. Below, we explore whether this behavior can be easily explained by accounts based solely on children's input for these expressions. These accounts would view PDDs and FRs as separate linguistic phenomena with no underlying commonality. As such, the observed simultaneous emergence of both constructions would need to be correlated with input frequency in some way, such that both constructions have similar frequencies in children's input.

Table 1 displays the results of a corpus analysis of child-directed speech portions of several naturalistic corpora from the American English section of the CHILDES database (MacWhinney 2000): Bates, Brown, Gleason, Hall, Kuczaj, Valian, VanHouten, and VanKleeck. This aggregated corpus contains 1,162,984 words and comprises speech directed at 198 children between the ages of 2 and 5 years old ${ }^{9}$.

Overall, the table shows a marked difference in overall frequency for the two relevant expressions (PDDs: 4749 vs. FRs: 606), which seems to immediately rule out a simple frequency-based account for explaining the observed simultaneous acquisition. However, it is possible that children might use frequency information in a more sophisticated manner.

\begin{tabular}{|c|c|c|}
\hline DPs & Definite-DPs & PDDs \\
\hline 138609 & 39179 & 4749 \\
\hline \hline WH-clauses & Embedded WH-clauses & FRs \\
\hline 29185 & 10548 & 606 \\
\hline
\end{tabular}

Table 1 Analysis of children's input. DPs = all determiner phrases, either singular or plural. PDDs = all plural DPs that are a definite description (i.e., they are prefaced by the definite determiner the $)$. WH-clauses = all clauses headed by a $w h$-word. Emb $\mathrm{WH}$-clauses $=$ all subordinate clauses headed by a $w h$-word. FRs $=$ all free relative clauses.

Another possible account, for instance, is that children learn the correct interpretation for PDDs and FRs simultaneously because they track the semantic interpretations of key words associated with these expressions, namely the for PDDs and $w h$-words like what for FRs. Whenever these key words are encountered, children observe whether the expression they are a part of - PDDs or FRs - refers to the maximal element of a given set. This account predicts that the is part of an expression associated with a maximal element as frequently as $w h$-words are part of an expression associated with a maximal element.

9 The breakdown of the input by age exhibits the same trend and is therefore omitted. 
We see from Table 1 that whenever the occurs at the beginning of a plural DP, the is part of an expression referring to a maximal element $100 \%$ of the time (4749 out of 4749). In contrast, $w h$-words are part of expressions referring to the maximal element of a given set much less often - only $606^{10}$ out of $29185(2.1 \%)$ or out of $10548(5.7 \%)$ if we restrict the relevant comparison set to embedded wh-clauses (since FRs are embedded $w h$-clauses). Clearly, the synchronized emergence of PDDs and FRs cannot be explained by an equal frequency of specific associated key words to denote maximal elements since these frequencies differ.

While we do not discount the possibility of some additional acquisition account based solely on input frequency that can explain the observed acquisition trajectory, the above accounts do not seem able to. These accounts viewed PDDs and FRs as separate linguistic phenomena with no real connection - both of them having a maximal interpretation was simply happenstance. As such, the only way to explain the simultaneous acquisition of the semantic interpretation was through similar input frequencies of some kind. The input frequencies for these expressions instead appear to be quite different. Given this, we believe it is more likely that something else is responsible for the synchronous emergence of the semantic representations for PDDs and FRs.

\section{Discussion: A linguistically-informed acquisition account and implications for semantic theory}

Our experimental findings show that children treat PDDs and FRs as semantically the same very early (4 years old), though they learn these expressions refer to the maximal element of a given set much later (6-7 years old). When we conducted a corpus analysis and examined several different acquisition accounts that viewed these expressions as unrelated, we found that none seemed capable of explaining children's observed acquisition behavior. The semantic analysis and the syntax/semantics mapping discussed in section 1 may offer a more viable explanation of these results.

Suppose that 4-year-olds master the compositional mechanism that is responsible for mapping the underlined NP in the DP the things on the plate onto the set of individuals. This assumption is supported by children being able to correctly understand other expressions containing that plural NP like the universally quantified DP all the things on the plate. Suppose also that they master the (different) compositional mechanism that is responsible for mapping the $\mathrm{CP}$ of a FR like what is on the plate

10 We note that the table presents an upper estimate on maximal FRs, since not all expressions matching the observable string pattern of FRs have a maximal interpretation, e.g. He went [FR where no one had gone before] (cf. Caponigro 2004 for additional discussion on this point). FRs using the wh-word what usually do not have this problem, so a conservative estimate of the FRs for this dataset would be 459. 
onto a set of individuals. Finally, suppose that children do not know that the overt definite determiner the in PDDs or the silent operator in FRs apply to those sets to return their maximal element, maybe because children do not know that there are sets of individuals containing plural individuals including the maximal element (we return to this issue below). Then, children would end up with a set-denoting expression as the argument of a predicate that selects for an individual-denoting expression - a semantic-type mismatch that could prevent the semantic computation from proceeding for both expressions ${ }^{11}$.

To solve this problem to PDDs and FRs, children may look for a solution that will work for both expressions, perhaps in the form of a semantic operation that will repair this type mismatch. Children could then look to the input for what this repair option might be and what semantic interpretation is associated with it. Informative data can come from either PDDs or FRs, since the same repair option with the same interpretation is associated with each.

This account predicts that children should have the same intuitions about the interpretations for PDDs and FRs at all ages - even if their intuitions are not correct because PDDs and FRs are connected by this common semantic representation issue. Our experimental results showed this: At every age, children seem to have identical interpretations for PDDs and FRs, even if their interpretations are not correct. This account also predicts simultaneous acquisition of the correct interpretation for these expressions, even if the input frequencies of these expressions are very different. Unlike the acquisition accounts considered in the previous section, this accords with our experimental results and corpus analysis.

But why do children not know before age 6 that the in PDDs and the silent operator in FRs are there to turn the set into its maximal element and therefore solve the type mismatch? Given the amount of input data available, we might expect them to acquire the correct interpretations earlier.

A possible answer concerns children's understanding of plural individuals. Huntley-Fenner (1995: Ch.4) demonstrates that 4-year-old children struggle with other expressions associated with the maximal element of a given set: collective nouns (e.g., they are unable to treat family as a countable unit, instead counting members of the family). We can imagine at least three hypotheses compatible with this behavior that bear on children's struggle with maximal individuals. First, perhaps children do not yet have the concept of plural individuals, which means they cannot

11 Children may then adopt various strategies to deal with this mismatch, not necessarily rooted in grammatical principles. Young children seem to treat PDDs and FRs the same as DPs with a nonsense determiner like blick. These are interesting issues that require further investigation. Our main concern here is just that the syntax/semantics mechanism in the grammar that children already master brings them to the same semantic conclusion: both PDDs and FRs denote a set of individuals and both trigger a type-mismatch. 
conceive of a maximal individual. Alternatively, they may know that there are plural individuals, but lack the knowledge that there are linguistic expressions that refer to those individuals. Thus, they would be unable to map any plural individual to a linguistic expression. Another option is that children's knowledge deficit is specifically about maximal individuals. They may not know that, given a set of individuals, the maximal element of that set is defined and there are linguistic expressions that refer to it. Each of these hypotheses should be tested separately, if possible, and predictions should be carefully investigated - and we leave that to future exploration.

In conclusion, we believe that our acquisition findings are both compatible with - and indeed support - the view that the meaning of PDDs and FRs is the same, resulting from similar general semantic principles. More broadly, we hope to have shown how experimental and corpus analysis techniques can inform semantic theory.

\section{References}

Abbott, Barbara. 2010. Reference. Oxford, UK: Oxford University Press.

Bernstein, Judy. 2003. The DP hypothesis: Identifying clausal properties in the nominal domain. In M. Baltin \& C. Collins (eds.), The handbook of contemporary syntactic theory, Oxford, UK: Blackwell.

Caponigro, Ivano. 2003. Free not to ask: On the semantics of free relatives and wh-words crosslinguistically: University of California, Los Angeles dissertation.

Caponigro, Ivano. 2004. The semantic contribution of Wh-words and type shifts: Evidence from free relatives crosslinguistically. In Robert B. Young (ed.), Proceedings from Semantic and Linguistic Theory (SALT) XIV, 38-55. Ithaca, NY: CLC Publications, Cornell University.

Chierchia, Gennaro. 1998. Reference to kinds across languages. Natural Language Semantics 6(4). 339-405. doi:10.1023/A:1008324218506.

Dayal, Veneeta. 1996. Locality in wh quantification. Dordrecht: Kluwer.

Dayal, Veneeta. 2004. Number marking and (in)definiteness in kind terms. Linguistics and Philosophy 27(4). 393-450. doi:10.1023/B:LING.0000024420.80324.67.

Flynn, Susan \& Claire Foley. 2004. On the developmental primacy of free relatives. MIT Working Papers in Linguistics 48. 59-69.

Gajewski, Jon. 2005. Neg-raising: Polarity and presupposition: MIT dissertation. Huntley-Fenner, Gavin. 1995. The representations of objects, non-solid substances, and collections in infancy and early childhood. Boston, MA: MIT dissertation.

Jacobson, Pauline. 1995. On the quantificational force of English free relatives. In Emmon Bach, Eloise Jelinek, Angelika Kratzer \& Barbara Partee (eds.), Quantification in natural languages, 451 - 486. Dordrecht: Kluwer.

Landman, Fred. 2000. Events and plurality. Dordrecht: Kluwer. 
On the acquisition of maximality

Link, Godehard. 1983. The logical analysis of plural and mass terms: A latticetheoretical approach. In Rainer Bäuerle, Christopher Schwarze \& Arnim von Stechow (eds.), Meaning, use, and interpretation of language, 302-323. Berlin: de Gruyter.

Löbner, Sebastian. 2000. Polarity in natural language: predication, quantification and negation in particular and characterizing sentences. Linguistics and Philosophy 23(3). 213-308. doi:10.1023/A:1005571202592.

Longobardi, Giuseppe. 2003. The structure of DPs: Some principles, parameters, and problems. In Mark Baltin \& Chris Collins (eds.), The handbook of contemporary syntactic theory, Oxford, UK: Blackwell.

MacWhinney, Brian. 2000. The childes project: Tools for analyzing talk. Mahwah, NJ: Lawrence Erlbaum Associates.

Modyanova, Nadya \& Ken Wexler. 2008. Maximal trouble in free relatives. In Harvey Chan, Heather Jacob \& Enkeleida Kapia (eds.), BUCLD 32: Proceedings of the 32nd annual Boston University Conference on Child Language Development, 287-298. Somerville, MA: Cascadilla Press.

Munn, Alan, Karen Miller \& Cristina Schmitt. 2006. Maximality and plurality in children's interpretation of definites. In David Bamman, Tatiana Magnitskaia \& Colleen Zaller (eds.), BUCLD 30: Proceedings of the 30th annual Boston University Conference on Child Language Development, 377-387. Somerville, MA: Cascadilla Press.

Partee, Barbara. 1986. Noun phrase interpretation and type-shifting principles. In Jeroen Groenendijk, Dick de Jongh, \& Martin Stokhof (eds.), Studies in discourse representation theory and the theory of generalized quantifier, 115143. Dordrecht: Foris.

van Riemsdijk, Henk. 2005. Free relatives: A syntactic case study. In Martin

Everaert \& Henk van Riemsdijk (eds.), The Blackwell companion to syntax, Oxford, UK: Blackwell.

Rullmann, Hotze. 1995. Maximality in the semantics of wh-constructions. Amherst, MA: University of Massachusetts dissertation.

Schwarzschild, Roger. 1996. Pluralities. Kluwer: Dordrecht.

Ivano Caponigro

Department of Linguistics

University of California, San Diego

9500 Gilman Drive, \# 0108

La Jolla, CA 92093-0108

ivano@ucsd.edu
Lisa Pearl

Department of Cognitive Sciences University of California, Irvine 3151 Social Science Plaza

Irvine, CA 92697-5100

lpearl@uci.edu 
I. Caponigro, L. Pearl, N. Brooks, and D. Barner

Neon Brooks

Department of Psychology

University of Chicago

5848 South University Ave.

Room Green 517

Chicago, IL 60637

neonblue@uchicago.edu
David Barner

Department of Psychology

University of California, San Diego

5336 McGill Hall

9500 Gilman Drive

La Jolla, CA 92093-0109

dbarner@ucsd.edu 